\title{
Cloud computing and web application-based remote real-time monitoring and data analysis: slurry injection case study, Onshore USA
}

\author{
Yonggui Guo $^{1}\left[\right.$ I $^{\prime} \cdot$ Ibrahim Mohamed $^{1} \cdot$ Omar Abou-Sayed $^{1} \cdot$ Ahmed Abou-Sayed $^{1}$
}

Received: 17 April 2018 / Accepted: 20 August 2018 / Published online: 24 August 2018

(c) The Author(s) 2018

\begin{abstract}
Remote live monitoring of field operations, such as injection, has been very restricted, although real-time data are often collected at field sites. The difficulties lie in the data access and limitations to obtain computing resources for data analysis, which restricts the engineers' abilities to provide useful and timely remote assessment and assurance to the operations. Cloud computing combined with web-based apps, however, makes it much easier and cheaper to monitor field operations in real time from anywhere around world. The current work provides our first attempt to apply the cloud computing and web-powered apps to monitor slurry injection at one injection site in Texas, USA. The site provides injection data that is stored automatically in a cloud database. The data are accessed and analyzed remotely through a web-based app in real time. Monitored injection pressure and rate provide the basis for pressure fall off analysis. If the fall off analysis yields an unanticipated fracture geometry, advanced 3D fracture simulations would be conducted to gain a better understanding of the effects of a specific injection on fracture geometry. The results of remote real-time data analysis set up early warnings to alert both onsite and offsite staff ahead of operational upsets. Compared to traditional desktop applications and isolated local data servers, cloud computing and web-based apps provide a more convenient and cost-effective way to monitor field operations in real time. The technique and workflow presented here may also be applicable to monitor other field operations.
\end{abstract}

Keywords Cloud computing $\cdot$ Web application $\cdot$ Remote real-time monitoring $\cdot$ Slurry injection

\section{Introduction}

Real-time monitoring has an important value in promoting an effective, safe, and efficient operation, particularly, for expensive offshore operations (BSEE 2016; Harder et al. 2015; Wang and Salehi 2015; Jahanbakhshi and Keshavarzi 2012; Dupriest and Koederitz 2005). For this reason, live data are usually collected and analyzed at field sites, but the use of real-time data remotely has not, yet, to become a standard practice in the oil and gas industries (BSEE 2014a, b; Van Oort et al. 2005; Laurens and Kales 2014). In fact, while most real-time monitoring work to date is done at field sites, only a small portion is conducted remotely, such as at remote Real-Time Monitoring Centers (RTMC) that are owned by either big operating companies or service contractors (BSEE 2014a).

Yonggui Guo

yguo@advantekwms.com

1 Advantek Waste Management Services LLC, 11000

Richmond Ave, Ste 190, Houston, TX 77042, USA
To access field data in real time at remote locations, remote real-time monitoring requires live data to be transmitted from field sites to a centralized data repository at frequencies ranging from seconds to minutes without serious delay. The data streams need to be saved to a dedicated data server for access by technical experts. Ideally, remote access must be possible from internet-enabled devices at other locations besides RTMC. In addition, an appropriate analytical software is also required to display the realtime data and perform specific analysis. For data-intensive analysis, a fast computer with adequate RAM is essential to ensure the analysis is completed in a timely manner. These requirements of massive data transmission, storage, access, and analysis limit the applications of remote real-time monitoring in oil and gas industries. Particularly, real-time data and/or analytical software often cannot be accessed from web-enabled devices; hence, currently remote real-time monitoring is done at fixed locations, such as RTMC, even though remote real-time monitoring capabilities have been built for field operations. 
Extensive and rapid advances in cloud computing allow users to access data, programs, and other computing resources from anywhere, at any time, and on any web-enabled device over the Internet. Cloud computing provides an optimal solution for the real-time data and/or software access problem. It also delivers on-demand service and offers a more flexible and cost-effective way to store data and obtain computing resources. Thus, it enables small operating companies to remotely monitor their operations in real time without a need for the dedicated network attached data servers and expensive RTMC typically required for remote real-time monitoring.

Although cloud computing is ideal for remote real-time monitoring, it alone is not sufficient to fully support the idea that remote real-time monitoring can be done on any webenabled device if conventional desktop applications have to be installed locally to facilitate data visualization and analysis (Borjas and Hamzah 2013). To avoid any inconvenience caused by the software installation, one approach is to view and analyze real-time data using web-based applications. Unlike their desktop counterparts, web-based applications are rendered and delivered through a web browser, and can be installed and run in a cloud environment. They are platform and hardware independent, and accessible from any web-enabled device connected to the internet. Since a web browser is the most often-used tool for end users, many conventional applications are being converted to web-based packages and migrated to the cloud to reduce cost and simplify the process of application management and update. The current paper reports on a novel application to access and analyze real-time injection data on any web-enabled device. This cloud-based application also facilitates remote real-time monitoring for injection.

\section{A new remote real-time monitoring model}

Although the importance of remote real-time monitoring has long been recognized by oil and gas industries, its applications to monitor field operations have been very limited. The typical existing remote real-time monitoring model is expensive and not affordable for small operators, because it requires a dedicated data server to store data and an onshore RTMC to monitor offshore operations remotely. A more cost-effective model has been developed to enhance remote live monitoring capabilities, as shown in Fig. 1. The novel concept utilizes several different ways to remotely monitor real-time data: (1) live data collected from each field site are processed through a Site Data Manager Server (SDMS) and then stored on a commercial cloud platform instead of a traditional local data server; (2) data are accessed, viewed, and analyzed through a cloud-based application instead of a conventional desktop application; (3) the cloud-based application is loaded on the commercial cloud platform rather than installed on local computers at RTMC; (4) data are accessible by any web-enabled devices, such as a hotel desktop or a smart phone, instead of only by computers at RTMC or company owned devices; (5) computing is done by cloud virtual machines rather than computers used to access the data at RTMC; (6) analysis results can be saved on either the cloud or local device; and (7) additional data can be loaded from any web-enabled device to the cloud through a web application to facilitate real-time monitoring/analysis.

Remote real-time monitoring requires live data to be collected at field sites, and then transmitted to a data server that is accessible by end users at any remote locations. In the past, data transmission has been one of the major bottlenecks for remote live monitoring. Several data communication technologies are available for real-time data transmission. For example, optical fiber, satellite, microwave, and
Fig. 1 Data collection, transmission, storage, access, display, and analysis model for remote real-time monitoring applied to Karnes injection project
Site Data Manager Server

Cloud-Based Applications (@SSURE)

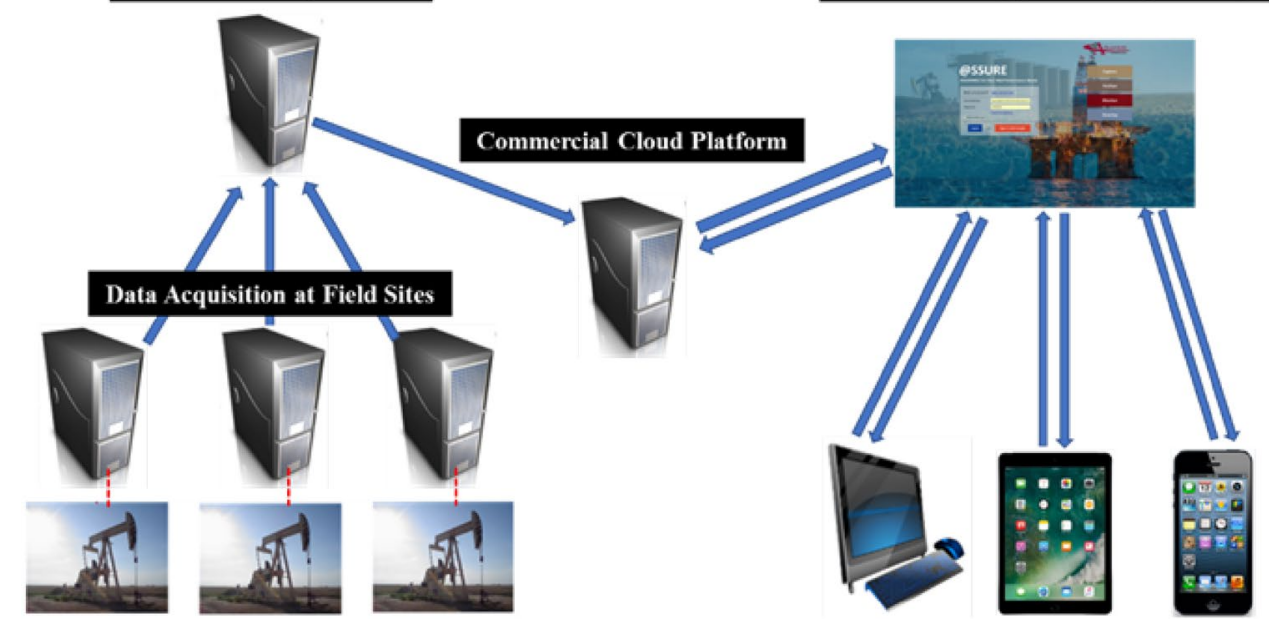


radiocommunication technologies have been used by the oil and gas industries for field operations (Black et al. 1997). The applicability of each technology to real-time monitoring is primarily dependent on its cost, bandwidth, and reliability. Improvements in telecommunications technology in recent years greatly expand data transmission boundaries and allow data transmission to remote locations at low cost, but in a fast and reliable way. For this reason, wireless technology is ideal to transmit data from field sites to commercial cloud platforms or dedicated data servers. Today, real-time data can be transmitted from our remote injection sites through a satellite wireless network without unacceptable latency at a cost of a few hundred dollars per month.

Data acquisition in the model is based on the wireless technology and done through a local computer that is connected to the sensors with wires. The computer is also connected to a local wireless Ethernet/IP network, so that the data can be transmitted to a remote SDMS. The data communication between the DMS and any field sites is based on a secure virtual private network (VPN), meaning that both the site computer and SDMS need to have an instance of VPN application running on them (Fig. 2). VPN running on the site computer is sending connection requests to a fixed IP address that is owned by SDMS, while VPN running on the SDMS is listening for incoming connection requests on a fixed port. Each site is identified by SDMS based on a client certificate generated by VPN for each site wireless router. The certificate is unique to the router.

For small-scale real-time monitoring projects, SDMS may serve only as a device manager and a data-processing center, although it can also serve as a data-storage center. This is primarily because it is not cost effective to maintain, manage, and backup a dedicated data server if it is not expected to receive and send a massive volume of data on a $24 / 7$ basis. Besides, stored data access from anywhere via internet connection could be a problem. Compared to the dedicated data server, cloud storage is not only cheaper, but also ideal for remote live monitoring because of fast and reliable data accessibility from anywhere of the world. For this reason, after sampling and processing the received data from a remote site, SDMS sends the data through internet to a commercial cloud platform (such as Microsoft Azure), although SDMS can also send the data to any customer's data server if it is preferred.

Besides data transmission and storage, another bottleneck for remote real-time monitoring is to access, view, and analyze data from any web-enabled devices, because conventional desktop applications must be installed on these devices to perform the task. To overcome the bottleneck, a cloud-based web application has been developed to facilitate remote real-time monitoring for injection (Fig. 3). Unlike desktop applications, the web application is delivered through a web browser. No installation is required to run the application.

The web application is a fully integrated data management, real-time monitoring, analysis, and assurance package delivered through Azure. It enables subsurface engineers to perform a broad range of analyses related to exploration, production, injection, and disposal processes based on both real-time data and stored data, such as log-based stress and rock property estimation, injection pressure falloff analysis, and injection-induced fracture simulation in 3D with an advanced fracture simulator (El-Fayoumi et al. 2011; Zaki et al. 2004). It provides stimulation, production, and injection engineers a common set of tools that they need to perform their typical workflows. In particular, the platform was developed to solve two particular pain points faced by engineers in today's oil and gas service and operating companies: (1) how to integrate various data types from distinct engineering tasks and to generate (and share) the workflow
Fig. 2 Data communication between field sites and SDMS. Real-time data collected from Karnes injection site is transmitted to SDMS through VPN

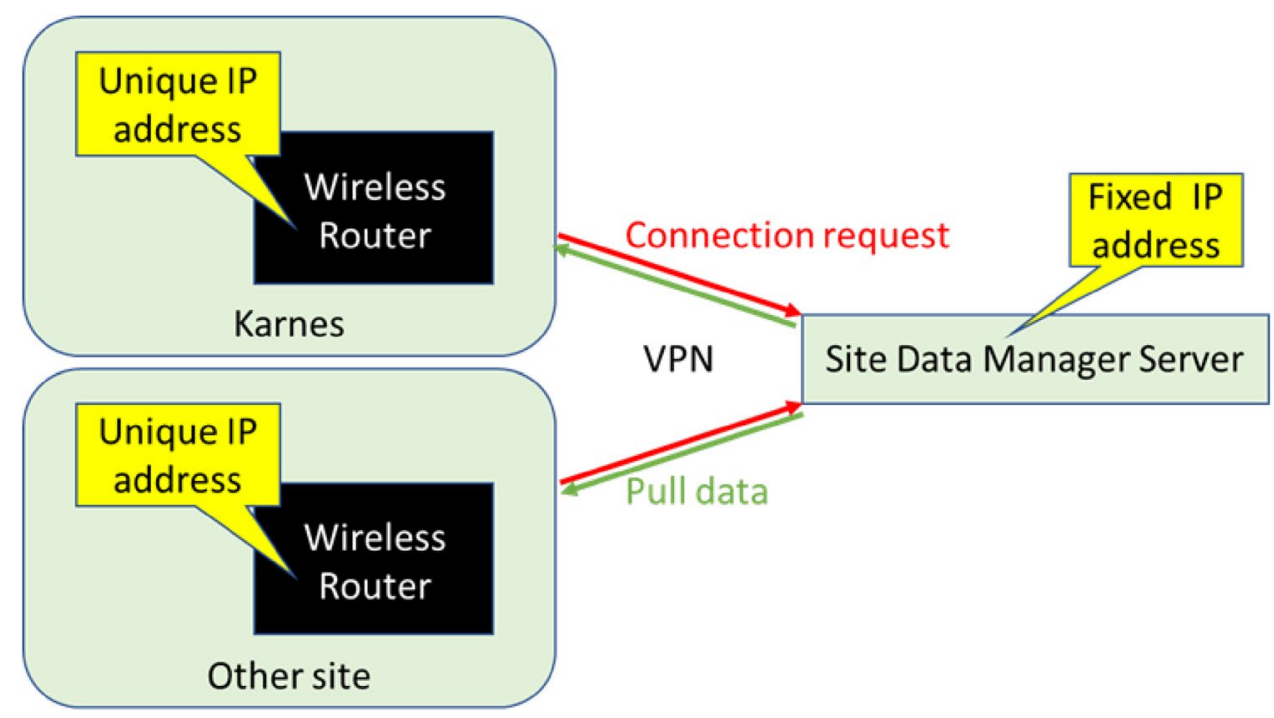


Fig. 3 Development of the web application in Azure. GUID stands for globally unique identifier. Red and blue GUIDs are analysis and data transmission requests, respectively. The application server handles all analysis requests. The data application sever processes all data transmission requests. The web server is a bridge that communicates between users and the other servers

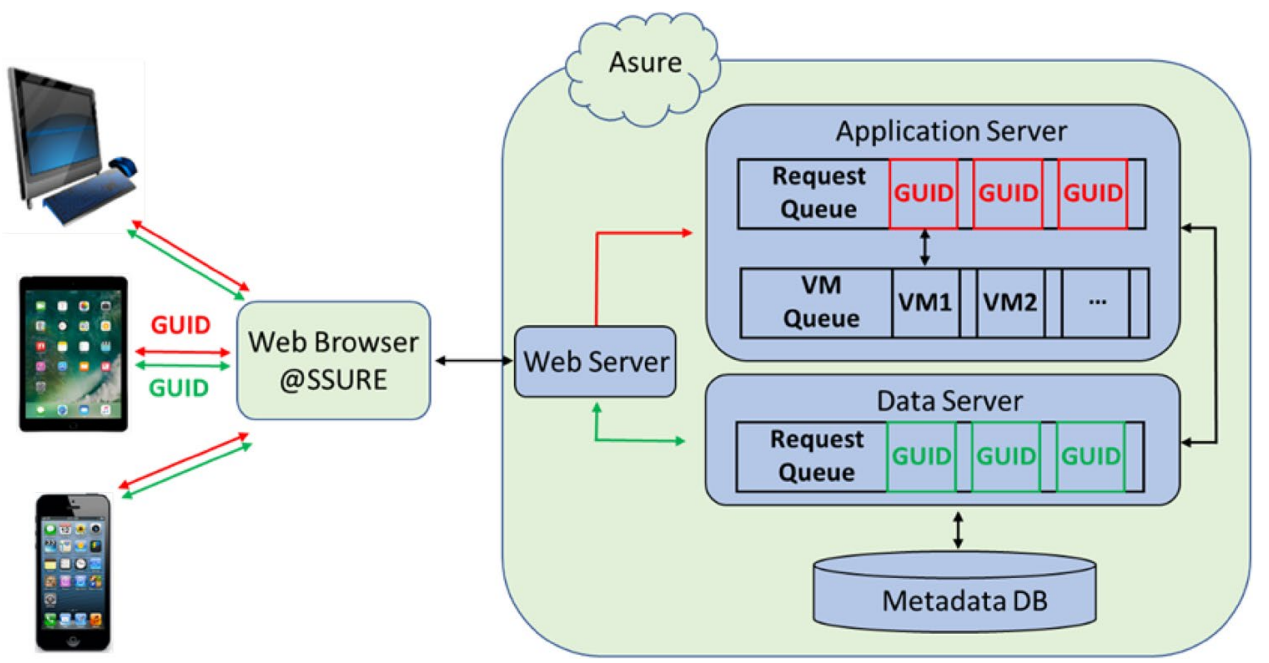

results in a framework that can be used by non-specialists from various locations and (2) how to access real-time data from wells at multiple field sites and execute, either by hand or automatically, detailed well performance analyses using best-in-class simulators (that are computationally intensive), so that changes can be made in the field before serious issues arise.

To accomplish these tasks in a time and cost-efficient way, a proprietary distributed architecture was needed using: (1) a commercial cloud platform (such as Microsoft Azure) or a customer's server behind a firewall that allows for distributed access, storage, and web service-based computation; (2) web service for running simulators and other executables on Azure/customer's server in batch and/or interactive modes; and (3) resilient and scalable back-end infrastructure based on client demand (both Azure and the web service computational and data needs on demand).

The details of the web application development strategy are illustrated in Fig. 3. The web application is accessible through a web browser from any web-enabled devices. It uses a globally unique identifier (GUID) to identify each user request that could be either an analysis request (red GUID) or a data transmission request (green GUID). The web server receives user requests, and sends received analysis and data transmission requests to the application server and the data server, respectively. The application server picks up items from request queue and assigns the job to a virtual machine (VM) with the lightest load. It launches each additional VM on Azure as needed. Because of this distributed computing infrastructure, the web application is capable of running simulations in parallel mode as well as running multiple simulations simultaneously by managing client demand and load balancing across multiple VMs.

The application server does not communicate directly with users. It only receives requests from the web server.
It pulls data required for each analysis from the data server. The data server receives requests from both the application server and the web server. Besides sending data to the application server, it also receives and stores all analysis status data and results. The web server, therefore, retrieves all analysis informations from the data server rather than the application server.

All components of the web application share an Azure data platform consisting of an SQL database (structured data tables), Cloud Tables (loosely associated data tables), and Blob Storage (blocks of unstructured data). The application database contains data from over 4 million wells, including well logs, reservoir properties, field measurements, drilling, completion, and production records. The majority of the data is saved in the Blob to reduce cost. Metadata are created and saved in the SQL database to help query and search through Cloud Tables and Blob Storage to find and locate a specific type of data in a time-efficient manner.

Since live data are stored on Azure, data transmission and storage are secured by Azure using industry-standard protocols. In addition, the web application is developed based on .NET Framework that provides a role-based security, i.e., it allows the application to make security decisions according to the identity or role membership of the user that interacts with the application. Users are required to have a user name and password to either fully or partially access the application and its linked database dependent on user authorization. To enhance data protection, regular users are not given permission to delete and rewrite any raw data, such as realtime injection data. An extra copy of entire database is also stored on Azure to ensure that the data are still available if the database is damaged or hacked.

There are many commercial cloud platforms suitable for the web application-based remote real-time monitoring and data analysis. The three major cloud providers, i.e., Amazon 


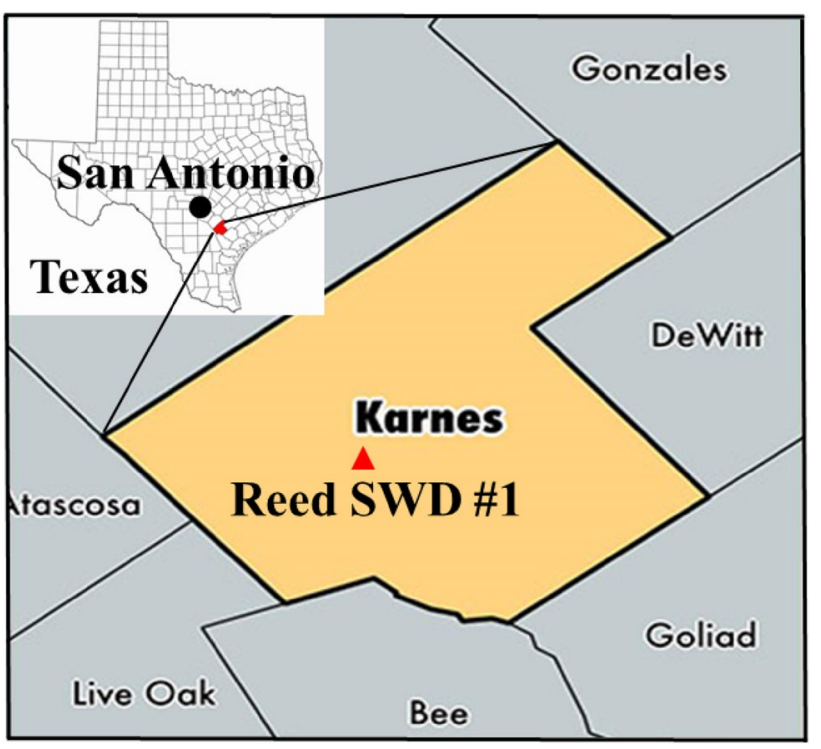

Fig. 4 Approximated location of Karnes injection site

AWS, Google Cloud, and Microsoft Azure, collectively dominate cloud storage and computing services. They offer comparable price to host our web application. Azure, however, provides more powerful and convenient tools to deploy and maintain the application as it is developed based on Microsoft ASP.NET and MSSQL database. We, therefore, deliver it through Azure.

\section{Slurry injection case study}

To dispose drilling waste through injection, an injection site was built in early 2015 in Karnes county-located approximately 50 miles southeast of San Antonio, Texas, USA (Fig. 4). A vertical disposal well (Reed SWD \#1) was drilled on site. The facility is capable of injecting up to $28,800 \mathrm{bbl} /$ day. The well, however, is permitted for injection at 25,000 bbl/day into the Upper Eocene Wilcox Sands (5970-7200 ft) with the maximum surface injection pressure of 2980 psi. GR and mud logs collected from the Reed SWD \#1 show that the Wilcox strata is composed of sandstone interbedded with shale layers. Its porosity and permeability measured from side wall core ranges from 18.5 to $25.9 \%$, and from 0.1 to $105 \mathrm{mD}$, respectively. The upper Wilcox strata is not extensively faulted. The well closest to the Reed SWD \#1 is a producer from a reservoir much deeper than the Wilcox formation. Their distance is more than 0.25 mile. The closest disposal well is about 1.8 mile away from the Reed SWD \#1. Its targeted injection reservoir is also within the Wilcox formation.

To meet the injection requirements for Texas, a sand interval from 6400 to $6655 \mathrm{ft}$ was selected for injection

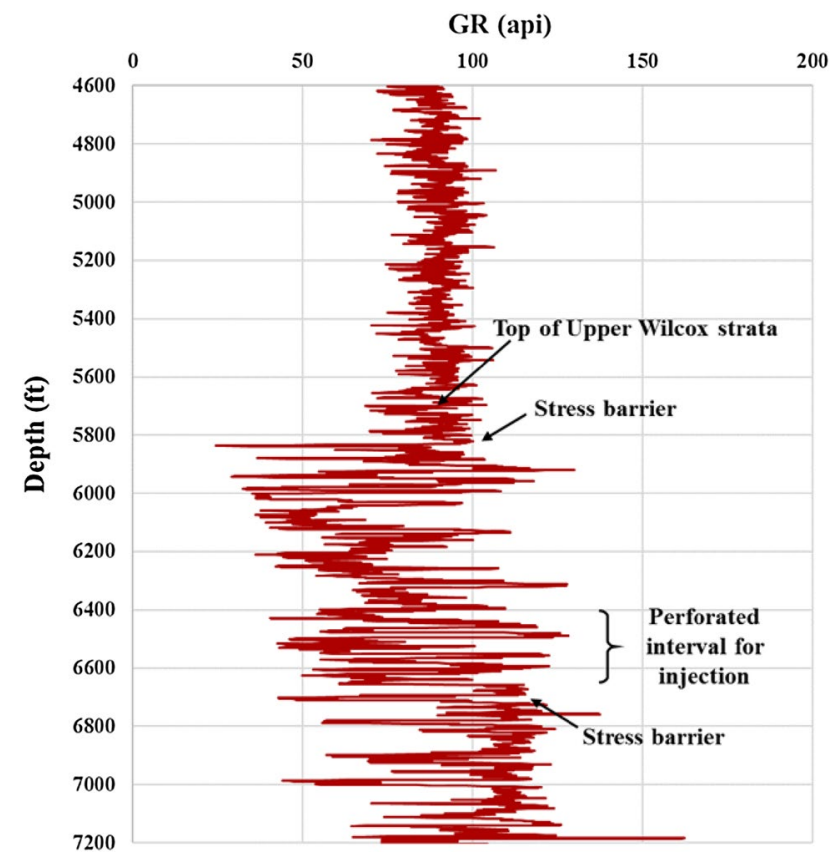

Fig. 5 Interval selected for injection. Gamma ray log was collected from Reed SWD \#1

based on regional injection experience, rock property, stresses, and injectivity analysis (Fig. 5). Log-based stress analysis indicates that thick shale layers above and below the injection interval are effective stress barriers to prevent injection-induced fracture growth out of targeted injection zone, which is consistent with fracture height prediction made by numerical models run under real injection conditions. The injection interval is slightly under-pressured. Pore pressure, suggested by drilling experience at about $2400 \mathrm{psi}$, is further confirmed by injection tests. The tests also show that interval breakdown pressure is about $5088 \mathrm{psi}$ (2385 psi surface pressure), while fracture propagation pressure is close to 4613 psi (1910 psi surface pressure). In addition, pressure fall off analysis indicates that fracture closure pressure is around $3850 \mathrm{psi}$ (995 psi surface pressure), and the interval permeability is less than $31 \mathrm{mD}$.

A thorough feasibility study was carried out for Karnes injection project and injection parameters were optimized based on injection test data and predictions from fracture simulations. Injection failures, however, could still occur during injection because of data uncertainties and the complex nature of injection (Bautista and Dahi Taleghani 2016; Mohamed et al. 2016). These potential injection risks may be related to unidentified pre-existing faults, overestimated barrier stress for fracture containment, plugging along the wellbore or in the fracture, inter-well fluid communication, well integrity issues (such as packer failure and poor cement job), and so on. Live injection data, however, would identify 
such risks at an early stage, so that mitigation actions are taken in a timely manner to minimize these risks.

Because of potential injection risks associated with well integrity and fracture containment, critical injection data (including injection rate, well head pressure, annulus pressure, slurry density, viscosity, and volume) are collected and monitored in real time on a daily basis to ensure that pressures, rates and other well performance metrics are behaving as expected. At Karnes injection site, the tubing pressure is measured by a pressure transmitter with an accuracy of $\pm 0.055 \%$ and a stability of $\pm 0.1 \%$ per 10 years. There is no downhole pressure gauge installed. Downhole pressure is estimated from the tubing pressure based on a power law fluid model for pipe flow (Govier and Aziz 1987). Commonly used Fanning friction correlations combined with viscometer readings taken at 300 and $600 \mathrm{rpm}$ during each batch injection are used to calculate friction loss. Injected fluid properties, such as density and viscosity, are measured during each batch injection. Injected fluid can be either water or slurry. Its density, therefore, varies from 8.3 to $12.0 \mathrm{ppg}$, while its viscometer readings at $300 \mathrm{rpm}$ and $600 \mathrm{rpm}$ range from 1 to 46 and 2 to 65 , respectively. The pumping rate of each pump is monitored by one flow meter with $\pm 0.75 \%$ accuracy of any indicated value. In addition, seven radar sensors are installed to take exact-level measurements of slurry and water tanks.

For the purpose of reducing the potential injection risks, Karnes injection activities have been monitored remotely in real time by well trained and experienced injection engineers at their offices and homes, and occasionally, also at preferred locations, where they have access to the internet. Real-time injection data, particularly, injection pressure and rate, are viewed and analyzed through the web application during each injection (Fig. 6). Real-time data are automatically loaded to the web application user interface if "live" data model is selected. Injection data of all the previous injection batches can also be displayed on four different time scales (i.e., hourly, daily, monthly, and yearly) for comparison and analysis. Instead of dedicated engineers monitoring the injection on a $24 / 7$ basis, user can create a custom alert or use default alert in the application, so that conditionbased real-time monitoring can be done. Once triggered, an alert sends an email and/or a text message to the user(s) and any engineer included in a pre-defined alert list, whenever real-time injection data do not meet default or user-defined

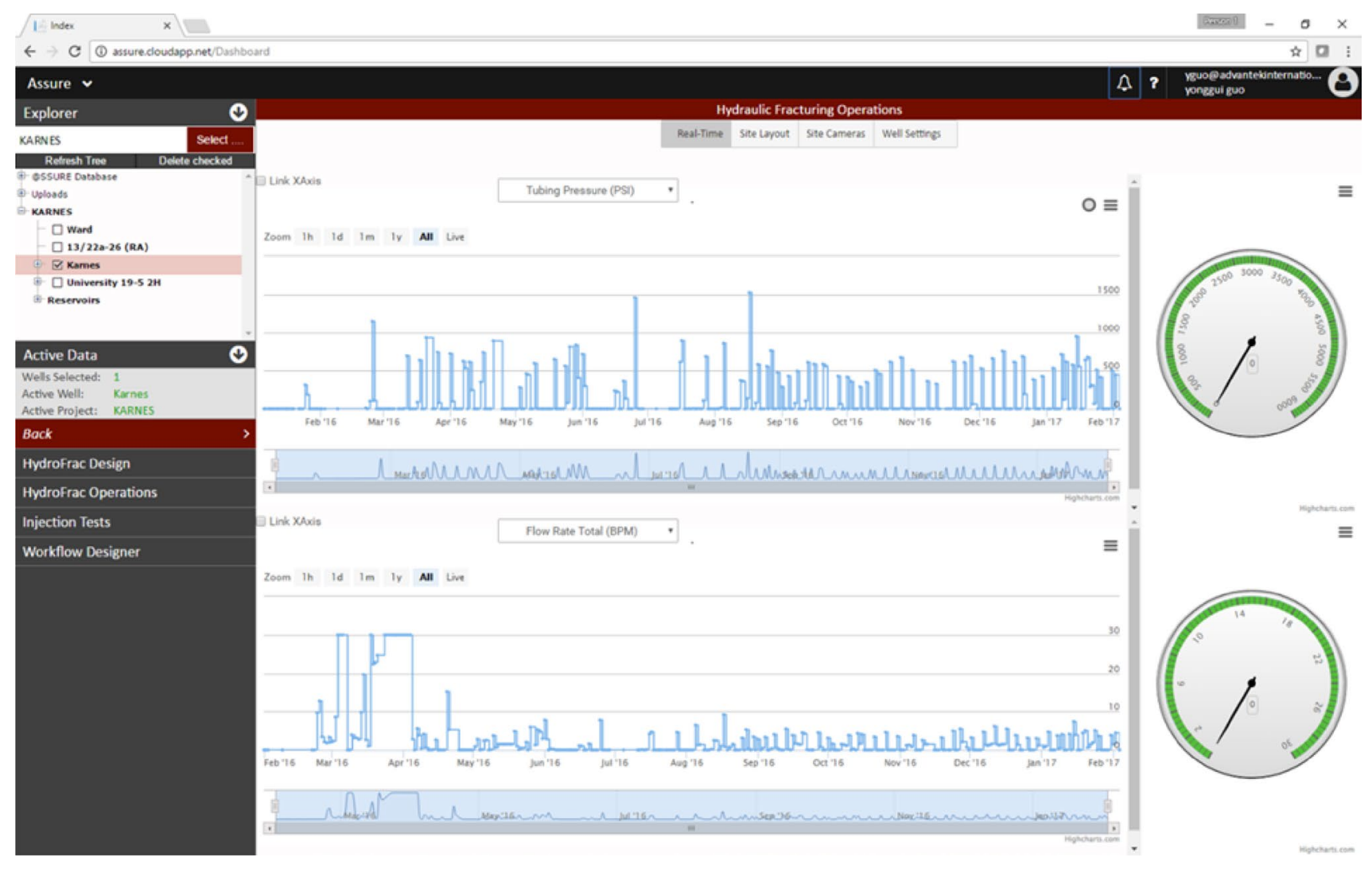

Fig. 6 User interface of the web application for remote real-time monitoring. Real-time injection data are loaded automatically from Azure. Note tubing pressure and flow rate plots only show rough variations of pressure and flow rate as they are down sampled significantly. The maximum tubing pressure during each injection is around 2000 psi 


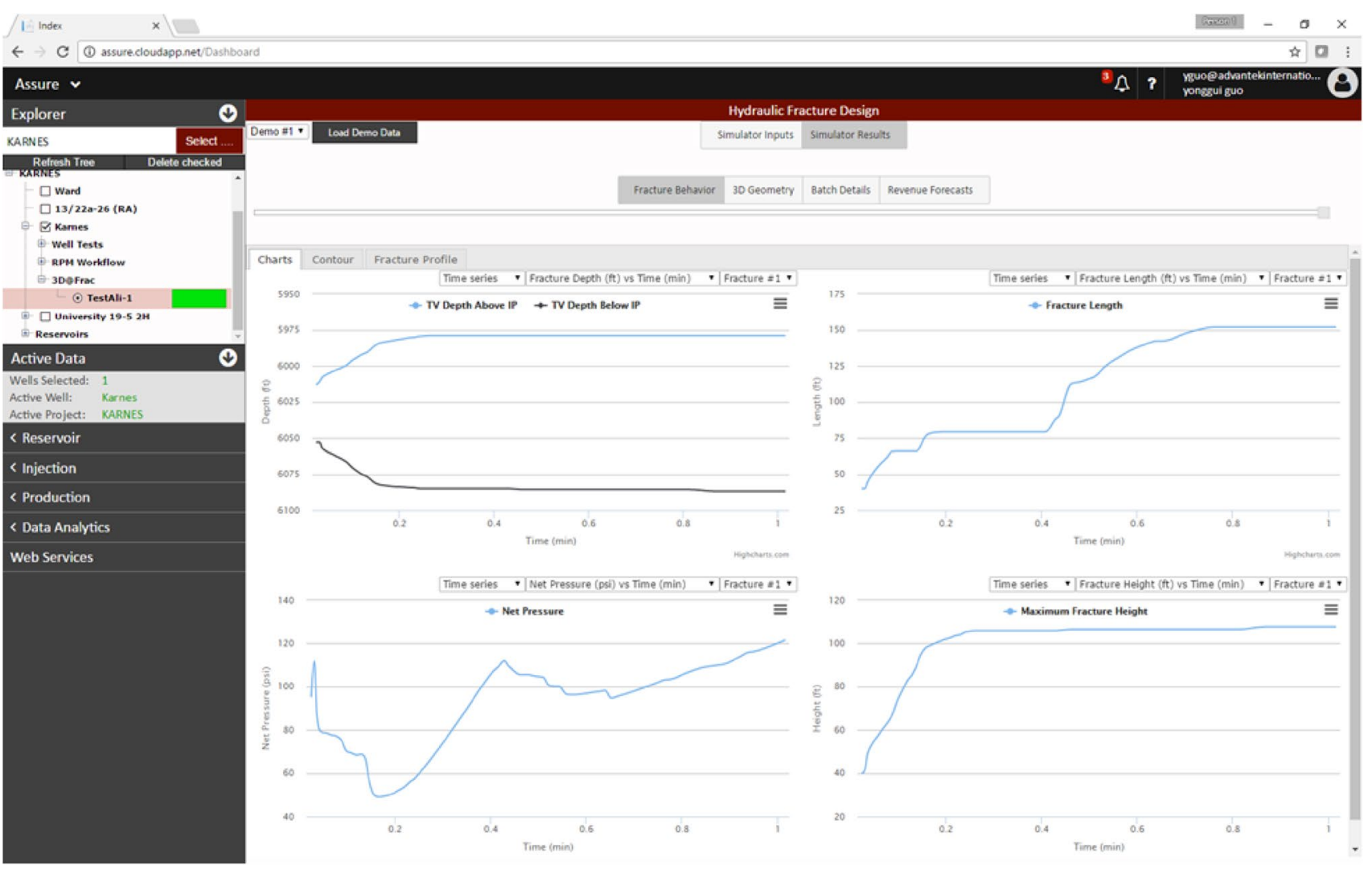

Fig. 7 User interface of the web application for 3D fracture simulation. Fracture simulations can be conducted before or during any injection under real injection condition, which provides valuable information about fracture geometry and it sensitivity to each injection parameter

Fig. 8 Geometry of fracture predicted by the $3 \mathrm{D}$ fracture simulator built in the web application. The simulations were run under real reservoir conditions for all possible injection scenarios. The results show that predicted fractures are contained within targeted injection zone. Higher solids concentration leads to shorter fractures, while higher injection rate results in longer fractures

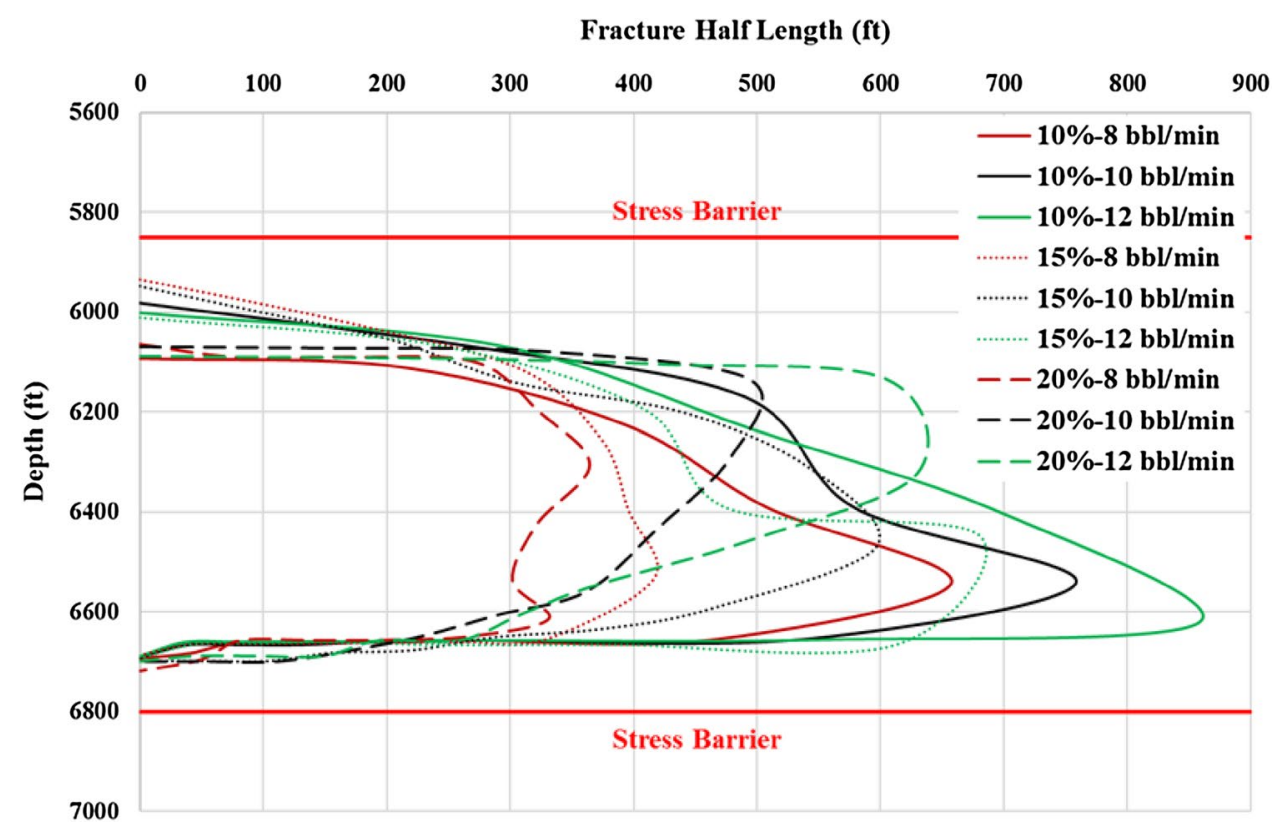

criteria, such as injection pressure exceeding user-defined limits or pressure variation deviating from user-defined trends.
Correct interpretation of real-time injection data requires some fundamental understanding of fracture growth within the injection zone. Hence, proactive 
monitoring is essential. Before any injection batch, 3D fracture simulations are conducted under real reservoir conditions to predict fracture containment, geometry, and its correlations with injection parameters (Fig. 7). For example, the 3D simulations show that for all possible injection scenarios, fractures are contained within targeted injection zone (Fig. 8). Fracture geometry is sensitive to slurry solid concentration and injection rate. Higher solid concentration leads to shorter fractures at a given injection rate, while higher injection rate results in longer fractures at a given solid concentration. The outcomes of the simulations are used to optimize injection parameters for next injection. They also help to optimize injection parameters during injection if undesirable injection data are observed. 3D simulations are also conducted during injection through the web application whenever there is a need to evaluate special injection cases to help interpret puzzling injection data.

Because the pressure falloff data of each patch injection after well shut-in provide valuable information about fracture geometry, injected zone properties, and stress state, a tool for pressure falloff analysis is built in the web application to enhance its capability for real-time injection data analysis (Fig. 9). After each batch injection, fracture half-length and width are estimated from pressure falloff analysis and compared with those predicted by the 3D fracture simulations. This step provides further assurance of fracture containment. Compared to log-based analysis, the pressure falloff analysis yields more reliable estimations of injection zone minimum stress and pore pressure, and their variations over time as a result of cyclic injection. Thus, the input parameters for the 3D fracture simulations can be tuned based on the results of pressure falloff analysis after each batch injection, which leads to a more reliable fracture prediction and a better understanding of injection operation effects on fracture geometry and spatial distribution.

3D fracture simulations combined with other calculations suggest that the highest possible solids disposal capacity of $2,312,752 \mathrm{bbl}$ may be achieved if slurry is injected at $10 \mathrm{bbl} /$ min with $15 \%$ solid concentration. To ensure the desired amount of solids can be injected into the targeted zone while keeping risk at a minimum, each batch injection was executed at an injection rate of $10 \mathrm{bbl} / \mathrm{min}$ with an averaged maximum solid volume concentration of $10 \%$ at the early stage of Karnes injection operation. Each batch includes a pre-flush of $500 \mathrm{bbl}$ water and a post-flush of $1000 \mathrm{bbl}$ water. Batch duration is $12 \mathrm{~h}$. The injection schedule worked well for first few injections. The maximum injection pressure of

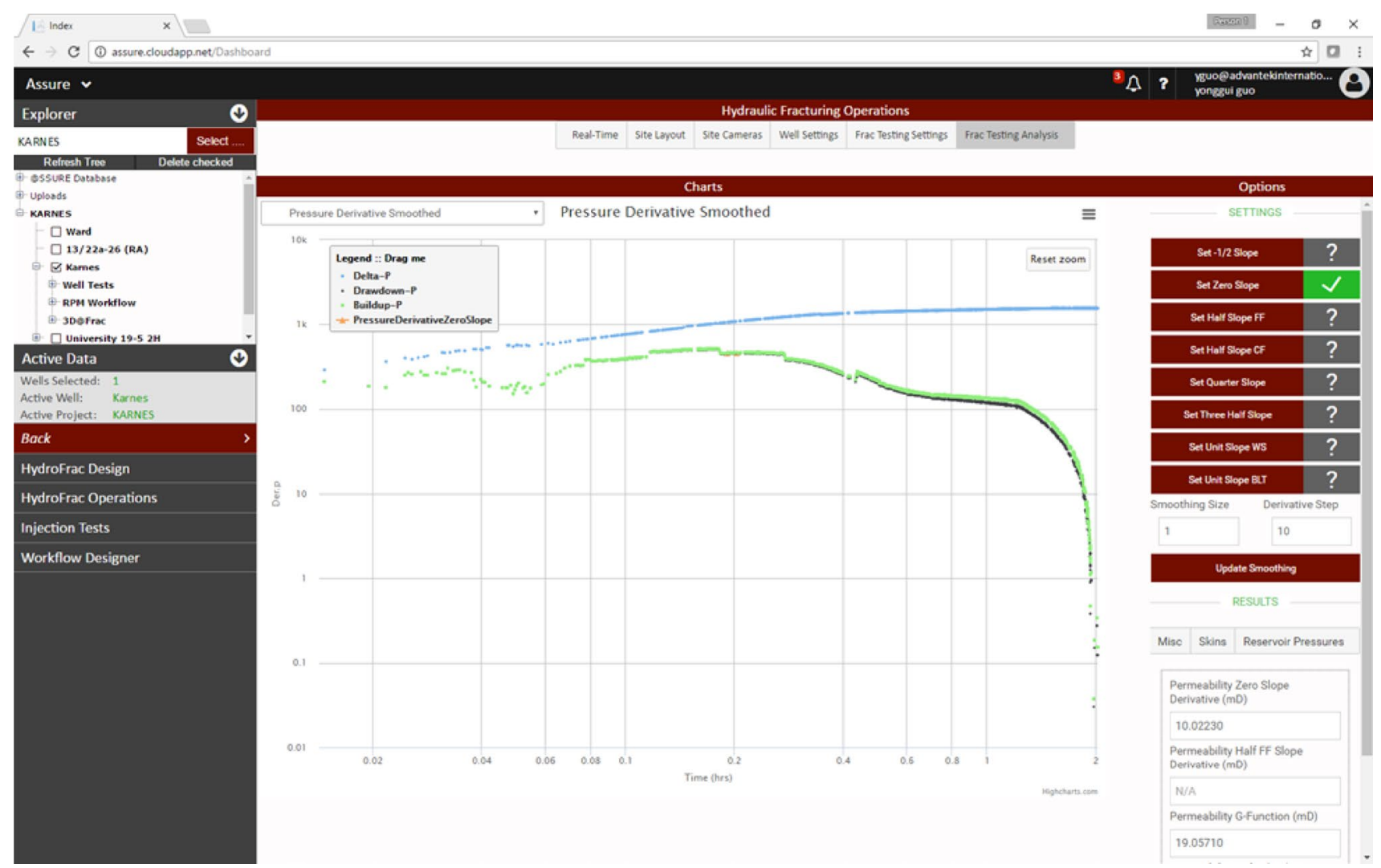

Fig. 9 User interface of the web application for pressure falloff analysis. The pressure falloff analysis is performed in an industry-standard way after each batch injection, which enhances the capability of the web application for real-time injection data analysis 
Fig. 10 Variation of the maximum injection pressure of each batch with injection rate. The increase of $2 \mathrm{bbl} / \mathrm{min}$ in injection rate keeps injection pressure below or slightly higher than 2100 psi for various solid volume concentrations up to $20 \%$

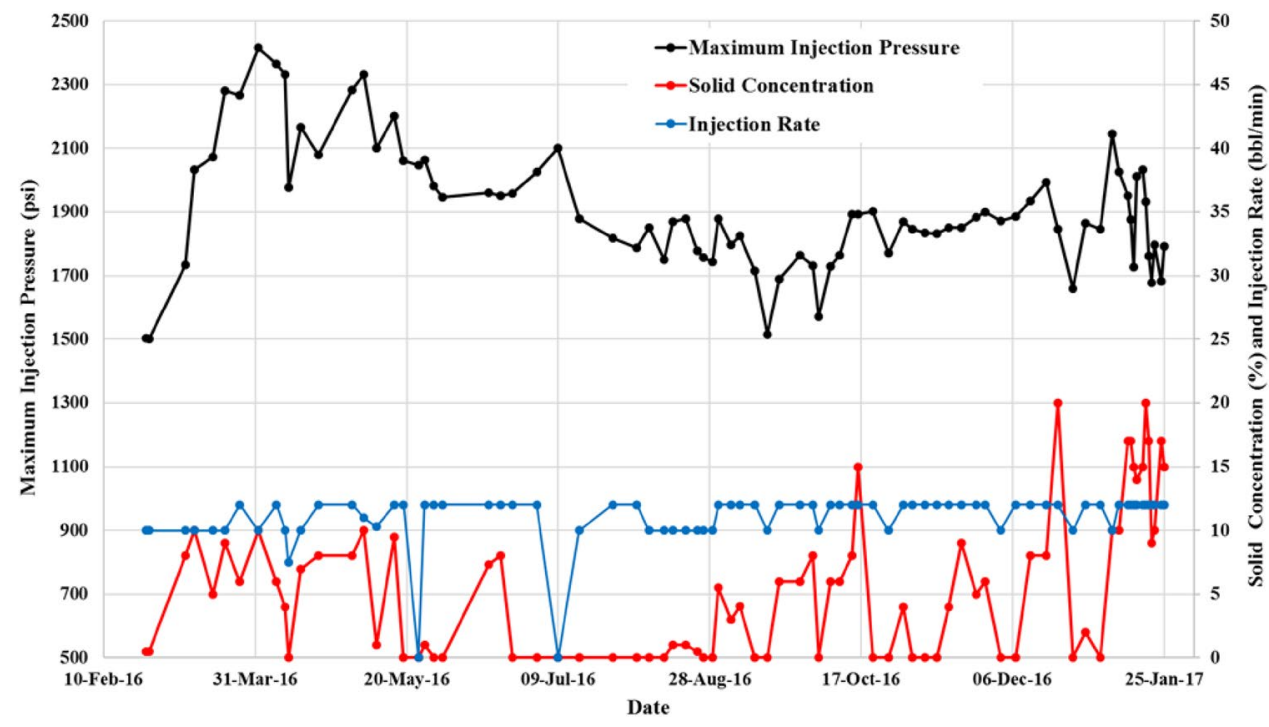

relatively poor understanding of the effect of slurry viscosity on fracture propagation.

To summarize, Karnes injection data are analyzed during and also after every batch injection to evaluate if injection parameters are optimal. Besides real-time injection data monitoring and evaluation to detect any abnormal injection pressure and pressure variation, the analysis also includes pressure fall off analysis and 3D fracture simulation mainly to estimate fracture geometry. In this way, we can assure fracture containment under preferred injection conditions and achieve expected injectivity. The analysis also helps us understand the effects of key injection parameters, such as how solid concentration controlled slurry viscosity and injection rate governed slurry shear rate affect the characteristics of injection pressure and injection-induced fracture, and what are critical pre-flush and post-flush volumes to avoid high injection pressure and minimize wellbore sedimentation and fracture plugging risks. A deep understanding of these effects allows us to react accordingly when abnormal injection pressure is observed from real-time injection data. As a full understanding of these effects may not be obtained from the analysis, experience is also critical to ensure that a correct decision is made. For this reason, offsite injection experts are also involved in the real-time monitoring. They remotely access the real-time injection data and help modify injection parameters whenever there is a need.

\section{Discussion}

As demonstrated by the monitoring work done for Karnes injection project, remote real-time monitoring is no longer a luxury service that only some major oil companies could provide for their high risk or deepwater operations. This is essentially because expensive RTMC is not a necessary besides it may also increase injection risks because of

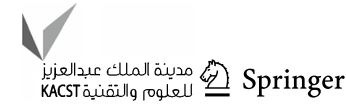


requirement for any remote real-time monitoring anymore. Live data can be transmitted, stored, and accessed through a commercial cloud platform that offers on-demand services. This is particularly beneficial for smaller operators and service providers as it is a cost effective alternative to a traditional data center. For example, our current cost for cloud storage and data transmission to support two injection projects is around $1200 \$$ per month. This is much lower than the cost (over $4000 \$$ per month) to maintain a local data server by a dedicated IT expert. In addition, cloud-based applications, such as the web application described in this paper, allow users to access real-time data and perform analysis with any web-enabled devices at any time from any location. Hence, remote real-time monitoring becomes more flexible and can be performed from any internet accessible locations rather than only at monitoring centers or fixed sites.

Although satellite internet does not provide super-fast data access, it is adequate to transmit injection data from remote injection sites to the cloud database in real time. This is because required data-transfer rate to push real-time data from an injection site to the cloud database is less than $1 \mathrm{~kb} / \mathrm{s}$. Except for numerical simulations of fracture propagation that may take hours, dependent on the complexity of subsurface structure and injected fluid properties, the computational time of all the other analyses is less than a few seconds. High-speed internet is required to view data and analysis results without latency as optimal data-transfer rate for the visualization can be more than $10 \mathrm{Mb} / \mathrm{s}$.

Overall cost for data storage, transmission, and analysis to remotely monitor the first injection site is around $1000 \$$ per month. Adding about $200 \$$ more allows each additional injection site to be monitored. The cost does not include internet expense of each injection site. Compared to onsite real-time monitoring, remote real-time monitoring is more cost effective to improve operation efficiency. This is because onsite real-time monitoring requires at least one highly experienced injection specialist at injection site during operation to view real-time data and make operation decisions. In addition, injection data that cannot be reviewed and analyzed remotely in real time limits quick collaboration between on-site personnel and remote subject matter experts when operational issues arise.

Unlike existing real-time monitoring models in which live data are saved on a dedicated data server, real-time data from Karnes injection site are stored on Azure (Fig. 3). This does not necessarily mean that the data must be installed on a commercial cloud to be effective. Storing Karnes injection data on a commercial cloud rather than a dedicated data server is indeed an economic decision rather than a technical decision. Note that the commercial clouds do not provide any special technique that is essential or preferable for the remote real-time monitoring. In fact, the only requirement for a data server to be suitable for remote real-time monitoring is that the data server needs to be accessible through internet at any time. Thus, the feasibility of the remote real-time monitoring is not affected if real-time data have to be stored on a dedicated data server because of data security or any other reasons.

Web-based applications, however, are essential for the remote live monitoring if the monitoring is expected to be done through any web-enabled devices. The major limitation for desktop applications built for real-time monitoring is that the applications have to be installed on a computer before using them. The installation requirement violates the concept of remote real-time monitoring, and restricts the availability and flexibility of remote monitoring through web-enabled devices. For example, remote monitoring may not be done through a hotel computer if the applications for monitoring cannot be installed. In contrast, web-based applications are installed on a remote server and accessible through a web browser. Because a web browser is a standard application for almost all web-enabled devices, it offers an ideal solution for the application accessibility problem. In addition, if data analysis is computational intensive, desktop applications may not be run efficiently if they are installed on a local device. In contrast, the efficiency of web-based applications is not affected by computational capacity of the local device as they are installed and run on cloud virtual machines. Although cloud virtual machines provide ondemand computing resources, the speed and efficiency of web applications may be further improved using emerging lightweight solutions, such as docker and kubernetes.

The web-based application is only employed to monitor injection in this study; the technique and workflow, however, can also be applied to monitor drilling, production, waterflooding, and other field operations. The fundamental difference among oil field operations in terms of remote real-time monitoring is that each operation is associated with a specific suite of data. Data types and analysis tools are usually different even for the same type of operations. Technically, a web-based application for remote real-time monitoring is essentially a data management platform. It has six key components, i.e., data collection, data transmission, data storage, data access, data display, and data analysis. From software development point of view, various data types can be easily built into an existing remote real-time monitoring platform, such as the web application presented here, but data analysis tools require the development of new functions. Although data analysis functions will need to be developed for a specific operation, the same remote real-time monitoring platform including the first five components can be shared by different operations if the platform is capable of accommodating all data types for these operations. With some minor modifications of such platform to include additional data types for different field operations, it may be 
applied directly to remotely monitor these operations if data analysis is not absolutely necessary.

\section{Conclusions}

Existing remote real-time monitoring technologies are often not economically feasible, leading to limited use of remote real-time monitoring systems by oil and gas industries. As telecommunication technology advances, field real-time data can be transmitted to a remote location in a more costeffective way. The data can be stored on either a commercial cloud platform or a dedicated data server that is accessible through internet. Compared to company owned data servers, cloud platforms not only provide almost unlimited date storage capacity at low cost, but also offer necessary computational power required for real-time data analysis. The combination of a cloud platform and web-based applications makes it more cost effectively to remotely monitor worldwide operations in real time.

Web-based applications are the ultimate way to take advantage of today's telecommunications and cloud technologies to enhance field operation efficiency. This is well demonstrated by the employment of the web application to remotely monitor injection in real time. Unlike traditional desktop applications, web-based applications fully support the concept of remote real-time monitoring, i.e., real-time monitoring is done through any web-enabled devices at anytime from anywhere in the world. With appropriate modifications, existing remote real time and data-driven monitoring platforms like the web application described in this paper are also applicable to other field operations, such as drilling and production.

Acknowledgements The authors would like to thank Advantek Waste Management Services LLC for permission to publish this paper.

Open Access This article is distributed under the terms of the Creative Commons Attribution 4.0 International License (http://creativeco mmons.org/licenses/by/4.0/), which permits unrestricted use, distribution, and reproduction in any medium, provided you give appropriate credit to the original author(s) and the source, provide a link to the Creative Commons license, and indicate if changes were made.

\section{References}

Bautista JF, Dahi Taleghani AA (2016) The state of the art and challenges in geomechanical modeling of injector wells: a review paper. ASME J Energy Resour Technol 139(1):012910-012910. https://doi.org/10.1115/1.4035257
Black DW, Tulissi M, Neudoerffer M (1997) Remote real-time monitoring and optimization of stimulation treatments using satellite technology. Pet Soc Can. https://doi.org/10.2118/97-104

Borjas R, Hamzah MK (2013) Bridging operational information with real-time data: enhancing real-time monitoring engineer's situational awareness. Society of Petroleum Engineers. https://doi. org/10.2118/167476-MS

BSEE (2014a) An assessment of the various types of real-time monitoring systems for offshore oil and gas operations (838 RTM report). https://www.bsee.gov/sites/bsee.gov/files/tap-technicalassessment-program/707aa.pdf. Accessed Jan 2017

BSEE (2014b) Summary of BSEE's real-time monitoring study. US Department of the Interior. http://onlinepubs.trb.org/onlinepubs/ sp//Cushing_Summary_of_BSEE_RTM_Study_March_2014.pdf. Accessed Jan 2017

BSEE (2016) Application of remote real-time monitoring to offshore oil and gas operations. https://www.bsee.gov/sites/bsee.gov/files /technology-assessment-program-tap/ab.pdf. Accessed Jan 2017

Dupriest FE, Koederitz WL (2005) Maximizing drill rates with realtime surveillance of mechanical specific energy, SPE paper no. 92194. SPE/IADC drilling conference, Amsterdam, 23-25 February

El-Fayoumi AM, Zaki KS, Abou-Sayed AS (2011) 3D hydraulic fracture simulation for injection in plastic shales. Society of Petroleum Engineers. https://doi.org/10.2118/142263-MS

Govier GW, Aziz K (1987) The flow of complex mixtures in pipes. Robert E. Krieger Publishing Co., Huntington

Harder C, Sutler J, Sawaryn S, Hale NW (2015) Real time data monitoring experience results in enhanced safety and efficiency, SPE/ IADC-173090-MS. In: Presented at the SPE/IADC drilling conference and exhibition, London, 17-19 March

Jahanbakhshi R, Keshavarzi R (2012) Real-time prediction of rate of penetration during drilling operation in oil and gas wells. In: 46th American rock mechanics/geomechanics symposium, Chicago, 24-27 June

Laurens M, Kales M (2014) Moving beyond realtime operations centres. In: Presented at the International petroleum technology conference, Doha, 19-22 January. https://doi.org/10.2523/IPTC17583-MS

Mohamed IM, Block GI, Abou-Sayed OA, Elkatatny SM, Abou-Sayed AS (2016) Flow rate-dependent skin in water disposal injection well. ASME J Energy Resour Technol 138(5):052906-052906. https://doi.org/10.1115/1.4033400

Van Oort E, Rosso RA, Cabello JE (2005) Evolution of real-time drilling operations: from concept and value justification to global implementation, SPE ATCE 97059. In: Presented at the SPE annual technical conference and exhibition, Dallas, 9-12 October

Wang Y, Salehi S (2015) Application of real-time field data to optimize drilling hydraulics using neural network approach. ASME J Energy Resour Technol 137(6):062903-062903. https://doi. org/10.1115/1.4030847

Zaki KS, Wang G, Meng F, Abou-Sayed AS (2004) A 3-D plastic fracture simulation to assess fracture volumes in compacting reservoir. American Rock Mechanics Association

Publisher's note Springer Nature remains neutral with regard to jurisdictional claims in published maps and institutional affiliations. 